Vol.01/ No. 01

Pages: $1-11$

https://www.irojournals.com/iroeea

DOI: https://doi.org/10.36548/jeea.2019.1.001

\title{
DEVELOPMENT OF AN EFFICIENT SMART GRID WITH ERROR CONTROL ALGORITHM
}

\author{
Rayudu Mannam \\ Vignan's University, \\ Chirra Engineers Pvt. Ltd. Bangalore 560062 \\ Email id: rayudu@chirra.in
}

\begin{abstract}
The in capabilities of the current power grid in distributing monitoring and managing due to the rising needs in the energy consumption and the aging infrastructure of the prevailing power grid, has led to the emergence of the grid that is smart in managing distributing and monitoring. The smart grid incorporating controls, computer, automation along innovative technologies and tools are able to provide an enhanced performance by being extra proficient, secure, reliable and greener. The smart grid also provides with the two way communication between the users and the utilities on the terms of electricity and information with the usage being completely monitored using the wireless sensor networks. The communication between the user and the utilities are often affected due to the noise, fading and path loss etc. experienced by the communication path. So the paper proffers an error control method that is adaptive to the changing behavior of the communication channels to make have an error less communication making the smart grid efficient. The result obtained based on the bit error rate, delay and the throughput evince the capability of the proposed scheme.
\end{abstract}

Keywords: Traditional power grid, smart power grid, error control coding, wireless sensor networks, secure transmissions, bit error rate, throughput and delay.

\section{INTRODUCTION}

The electric grids that are available today were proffered hundreds of years ago when the necessity for the electricity was very meager and the utilization was simple as the power utilization at homes were limited to one or two lights and fans. The electric grid was also designed to meet the limited utilization with the bill filed regularly on the bases of the usage per month. This conventional power grid was a one way communication from the providers to the users causing difficulties for the grid to respond to the growing necessities of the $21^{\text {st }}$ century. The increasing electrical and electronic appliances have also increased the need for the electricity supply at homes and the industries. This entailment in meeting the rising requirements and the aging infrastructure of the current grid scenario and elude the issues related to the maintenance along with the scalability and the lack of efficient management leading to a potential failures[11] so there elevates a need for a better reliable grid to provide an enhanced efficiencies in the functioning and the customer service [6] and Smart grid paves way for automated energy supply that could be 
Journal of Electrical Engineering and Automation (EEA) (2019)

Vol.01/ No. 01

Pages: $1-11$

https://www.irojournals.com/iroeea

DOI: https://doi.org/10.36548/jeea.2019.1.001

distributed over wider area [4] it is equipped with proficiency of handling the challenges incurred in the current power grid [7] The smart grid also acts a solutions to the transformation occurred in the technologies towards sustainability by adopting to the distributed generation and the micro grids [8] the smart grids include the renewable energy sources such as the wind mill and the solar panels and other technologies that are modernized (smart meters, smart sensing and communicating devices) to be successful in the addressing the requirements as well as managing the systems. The fig. 1 below shows the traditional grid and the smart grid.

Traditional power grid

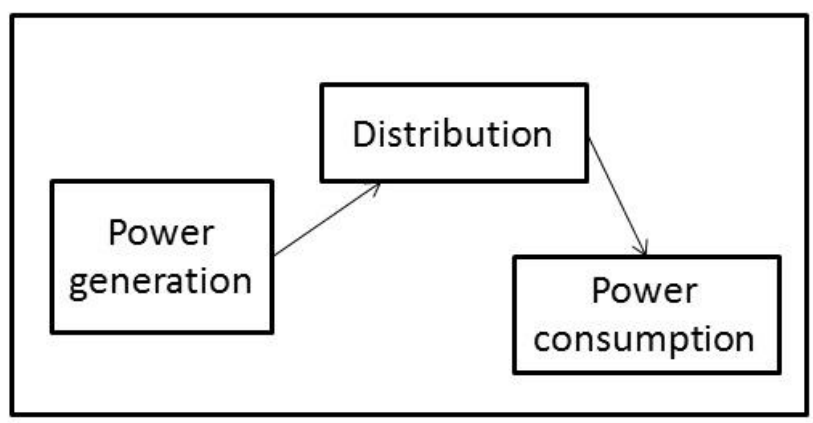

Smart power grid

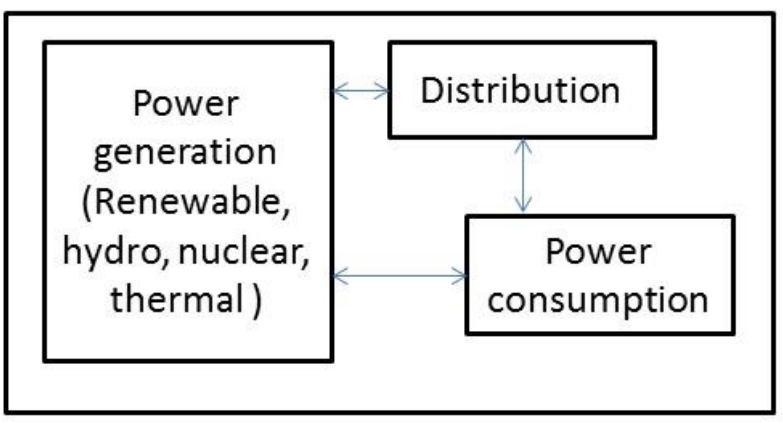

Fig .1 Traditional and Smart Power Grids

The bidirectional communication enabled by the smart grid, enables the user to monitor their daily consumption and reduce their electricity charges, enables the users to prioritize their works and limit theirs usage on the time of the heavy consumption. They enable to have an efficient distribution of the electricity with the a faster restoration, reduced peak demands, operations and the management costs and further increases the renewable energy systems to a large scale. The tabulation below in the table 1 shows the characteristics of the traditional and the smart grid as gathered from the [4] and [8] 
Journal of Electrical Engineering and Automation (EEA) (2019)

Vol.01/ No. 01

Pages: $1-11$

https://www.irojournals.com/iroeea

DOI: https://doi.org/10.36548/jeea.2019.1.001

\begin{tabular}{|l|l|}
\hline \multicolumn{1}{|c|}{ Traditional Grid } & \multicolumn{1}{c|}{ Smart Grid } \\
\hline They are electromechanical & $\begin{array}{l}\text { It is a electrical network in } \\
\text { combination with the digital } \\
\text { communication }\end{array}$ \\
\hline Provides one way communication & Allows two way interaction \\
\hline $\begin{array}{l}\text { It is a centralized generation with few } \\
\text { sensors, lags automatic monitoring }\end{array}$ & $\begin{array}{l}\text { The generation of the power is } \\
\text { distributed, as it allows an easy } \\
\text { integration of variety of power } \\
\text { generation sources }\end{array}$ \\
\hline $\begin{array}{l}\text { Does a manual monitoring with manual } \\
\text { recovery }\end{array}$ & $\begin{array}{l}\text { Does an automated monitoring and } \\
\text { recovery }\end{array}$ \\
\hline $\begin{array}{l}\text { Concurrent production and } \\
\text { consumption of energy and electricity }\end{array}$ & $\begin{array}{l}\text { The power generated are accumulated } \\
\text { using the storage system }\end{array}$ \\
\hline Responds slowly to exigent situations & $\begin{array}{l}\text { Responds immediately on the cases of } \\
\text { the emergencies }\end{array}$ \\
\hline Avails only limited customer choices & Avails extensive choices for the users \\
\hline
\end{tabular}

Table. 1 The characteristics of the Traditional and the Smart Grid

Despites it capabilities, the smart grid suffers from a certain issues based on the security, as they monitored information's being transmitted are liable of being affected by the errors that occurs due to the path fading path loss and noises etc. so there arises a need for the error controlling mechanism to eradicate the problems arising due to the error in the communication of the information's.

So the paper proffers an error control algorithm that is adaptive based on the hash-Reed-Solomon method to elude error occurrence in the information transmission between the users and the utilities and the energy providers.

The paper remaining is organized with the 2. Related works in on the smart grids and the issues in communicating, 3. The proposed work of hash-Reed-Solomon error control algorithm that is adaptive, 4. Result evaluation on different modulation schemes, 5. Conclusion 
Journal of Electrical Engineering and Automation (EEA) (2019)

Vol.01/ No. 01

Pages: $1-11$

https://www.irojournals.com/iroeea

DOI: https://doi.org/10.36548/jeea.2019.1.001

\section{RELATED WORKS}

Smart grid emerged as a novel technology to manage the electric supply and reduce the electricity consumption is employed in many applications including the cellular network, an energy efficient smart grid utilizing the renewable resources making the communication greener is presented in $\mathrm{Bu}$ et al [1] along with the open issues on interactive sessions for the smart grid infrastructure in the Erol-Kantarci, et al [2]. The smart grid seeming to be very enhancement of the present day grids, also serve as promising technology in helping the users to reduce the energy consumption, has revamped the electricity generation and the supply. The path leading to the energy supply based on the concept of the smart grid is proposed in Farhangi et al [3]. The capability of the smart grid are explored in the following papers of Fang et al [4] who presents the survey of the smart gird as a smart management, and protection system by exploring its infrastructures, subsystems and the information subsystems that are smart, Amin et al [5], Ipakchi et al [6] details the essentialities of the smart grid as the secure, intelligent, fast, accurate and distributed. Gungor et al [7] scopes in detailing the present day scenario with the smart grid and further discusses the challenges related to the research in it. Tuballa, et al [8] reviews the smart grid by surveying the functionalities, its characteristics, and features, further details the improved supply based on the demand using the smart grid. Kabalci et al [9] the paper describes the clear point about what are smart grids and the communication methods used. The security requirements of the smart grid are also surveyed in this paper. Kim et al [10] and, Kim et al [12] the paper aims in reducing the influence of the impulsive noise and the channel attenuation, in the power line communication system by clipping and equalizing. Fadel, et al [11] the paper elaborates the WSN for the smart grid applications Narendran et al [13] the paper presents the unified power control performance and the forward error coding algorithm for the enhancing the system capacity and reducing the power utilization for the mobile users.

\section{PROPOSED WORK}

The smart grid employing and engaging multitude of innovative technologies, to automate the distribution of the system and avail many choices for its users involves components like, smart sensors and smart meters to enable the user to reduce the unwanted usage and limit the electricity bill by communicating in a smart way, it does an overall monitoring enabling the customers to prioritize their needs and reduce their demands on the time of peak consumption, the regularize their flow finding an alternative way if any fault is found in the substation in between. Thus they meet the increasing demands in the intelligent way. Despite their merits the smart grids often faces issues in the communication of the data monitored. The usage of the wireless medium in communication of the information monitored, is often introduces difficulties in transmitting the information monitored as they are liable of being affected by path loss, error occurrence and the path fading. This affects the two way communication which is the essential characteristic of the smart grid. So the proffered method utilizes the hash-Reed-Solomon method in error 
Journal of Electrical Engineering and Automation (EEA) (2019)

Vol.01/ No. 01

Pages: $1-11$

https://www.irojournals.com/iroeea

DOI: https://doi.org/10.36548/jeea.2019.1.001

control, the algorithm is adaptive to the changing bandwidth and the transmission behaviors of the communication channel. The fig. 2 below shows a block diagram of the proposed method in the smart grid.

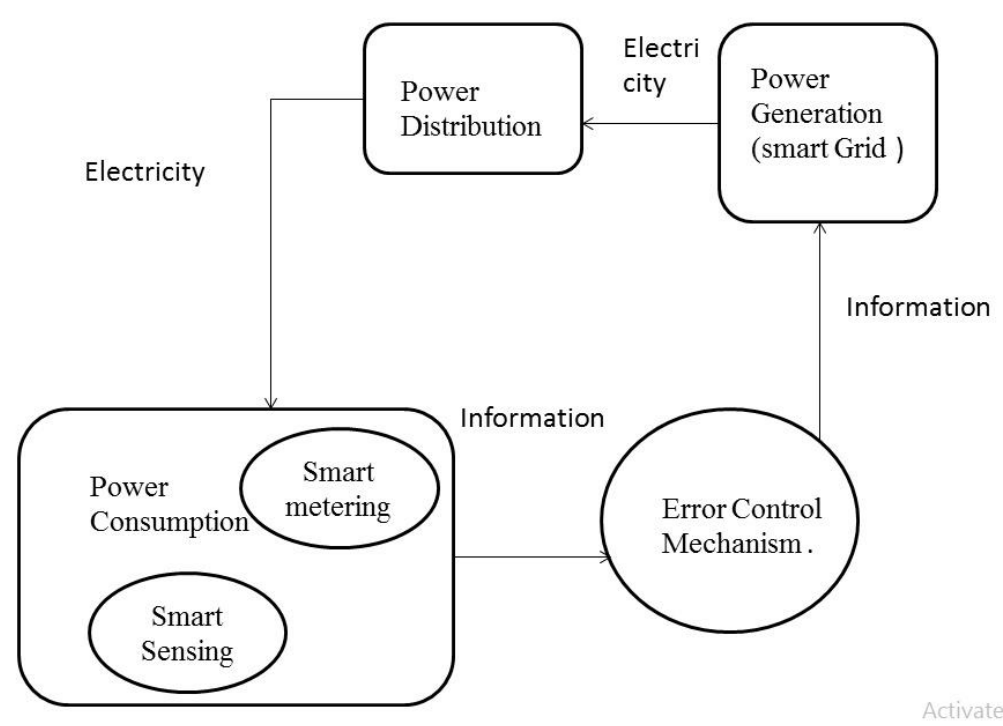

Fig .2 Error control in the Smart Grid

The fig . 2 shows the incorporation of the Hash-Reed-Solomon algorithm in the smart grid, to overcome the security issues prevailing in the communicating of the monitored information's allowing a two way interaction possible and making the smart grid efficient.

\subsection{HASH-REED-SOLOMON ADAPTIVE ALGORITHM FOR ERROR CONTROL (HRS- AAEC)}

The proffered system utilizing the HRS-AACE is proceeds taking triple leaps as, assigning of the Reed-Solomon hashes, to the wireless- nodes involved in the monitoring and communicating, establishment of the updation table and swapping among the Reed-Solomon hashes based on the verges set.

The initial step with the establishment of the updation table, utilizes the greedy method in deciding with the optimal Reed-Solomon hashes for the provision of the enhanced solutions for the smart grid. The hashes of the Reed - 
Journal of Electrical Engineering and Automation (EEA) (2019)

Vol.01/ No. 01

Pages: $1-11$

https://www.irojournals.com/iroeea

DOI: https://doi.org/10.36548/jeea.2019.1.001

Solomon contains two parts the data part assigned as ' $d$ ' and the parity part assigned as the ' $2 p$ ' and the total length of the hash is assigned as ' $N$ ' the parity bit takes the length of the $N-k$ which is equal to ' $2 p^{\prime}$. The fig.3 below shows the hash for the Reed-Solomon.

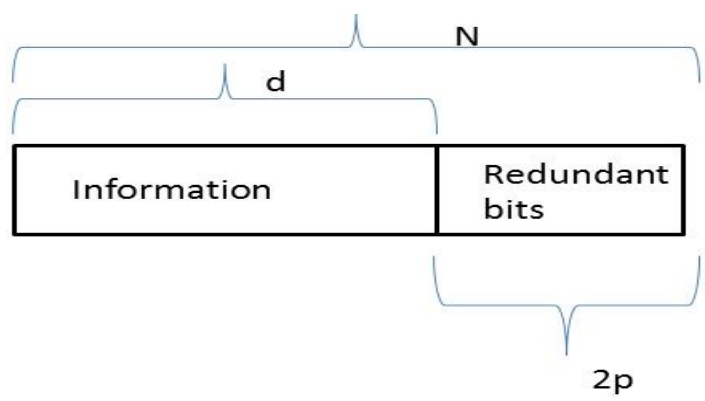

Fig .3 Has-Reed-Solomon Properties

The proposed method utilizing Reeds-Solomon employs certain hashes such as the $R S H(40,36)$ $R S H(46,36), R S H(51,36), R S H(58,36)$, and $R S H(64,36)$. The se hashes are assigned to the respective wireless nodes engaged in the monitoring to according to the distance from the substations, so the assigning of the RSH proceeds with the enumeration of the length between the wireless-nodes and the substations, and the normalization of the length and the higher normalization code were assigned with the higher hash values with higher redundant bits and the lower code value were assigned with the lower hash values and lower redundant bits. Now the RSH are assigned to the each wireless-nodes based on the distance from the base station.

The next step proceeds with the updation of the table with the based on the bit error rate ranges and the assigning of the $R S H$ vary based on the channels behavior and the $R S H$ optimal for the channel behavior was enumerated based on their solving capabilities of the bit error rate ranges and assigned to the channel to provide with a successful communication.

Later the $R S H$ assigned to the particular channel enumerated based on the bit error rate ranges are swapped with the higher hash values with higher redundant bits if the error occurrence was more and swapped with lower hash values with lower redundant bits if the error occurrence was less. The error occurrence rate was estimated by setting a verge level for the occurrence of the error. The algorithm below in the fig. 4 gives the steps in the error control for the secure transmission of information. 
Journal of Electrical Engineering and Automation (EEA) (2019)

Vol.01/ No. 01

Pages: $1-11$

https://www.irojournals.com/iroeea

DOI: https://doi.org/10.36548/jeea.2019.1.001

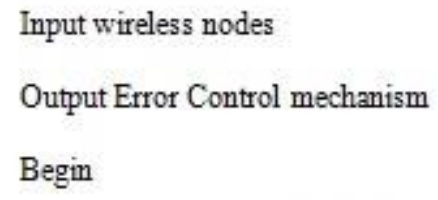

Fig .4 Algorithm for Error control in Smart Grid

\section{RESULTS}

The evaluation of the smart grid utilizing the hash-Reed-Solomon method to establish an error control method that is adaptive according to the changing channel behavior based on the error occurrence is validated with different modulation schemes, with minimum number sensor nodes ranging within hundred over an area of $200 * 200 \mathrm{~m}^{2}$ with a bit error rate of the $10^{-4} \leq$ bit error rate $<10^{-2}$, over a number of channels ranging within 8 , the table. 2 below shows the updation table for the channels ranging within 1 to 8 and the decision of the optimal $R S H$ based on the bit error rates for a substation of range $200 \mathrm{kV}$. 
Journal of Electrical Engineering and Automation (EEA) (2019)

Vol.01/ No. 01

Pages: $1-11$

https://www.irojournals.com/iroeea

DOI: https://doi.org/10.36548/jeea.2019.1.001

\begin{tabular}{|c|c|c|}
\hline RSH & Bit Error Rate & Optimal RS code \\
\hline RSH $(40,36)$ & $10^{\wedge}-3 \leq \mathrm{BER} \leq 1$ channel 1 & $\operatorname{RSH}(64,36)$ \\
\hline RSH $(40,36)$ & $10^{\wedge}-4 \leq \mathrm{BER} \leq 10^{\wedge}-2$ channel 8 & $\operatorname{RSH}(58,36)$ \\
\hline RSH $(46,36)$ & $10^{\wedge}-4 \leq \mathrm{BER} \leq 1$ channel 1 & $\operatorname{RSH}(52,36)$ \\
\hline RSH(46,36) & $10^{\wedge}-8 \leq \mathrm{BER} \leq 10^{\wedge}-6$ Channel 8 & $\operatorname{RSH}(58,36)$ \\
\hline $\operatorname{RSH}(52,36)$ & $10^{\wedge}-11 \leq \mathrm{BER} \leq 10^{\wedge}-9$ channel 1 & $\operatorname{RSH}(58,36)$ \\
\hline RSH(52,36) & $10^{\wedge}-12 \leq \mathrm{BER} \leq 10^{\wedge}-9$ channel 8 & $\operatorname{RSH}(64,36)$ \\
\hline RSH(58,36) & $0 \leq \mathrm{BER} \leq 1$ Channel 1 and 8 & RSH(64,36) \\
\hline RSH(64,36) & $0 \leq \mathrm{BER} \leq 1$ Channel 1 and 8 & $\mathrm{RSH}(64,36)$ \\
\hline
\end{tabular}

Table2. Updation Table

The application of the error control in the smart grid in the process of the information transmission enables the conveyance of the information without delay. Throughput achieved ensures the successful delivery of the information's compared to the channel without error control.

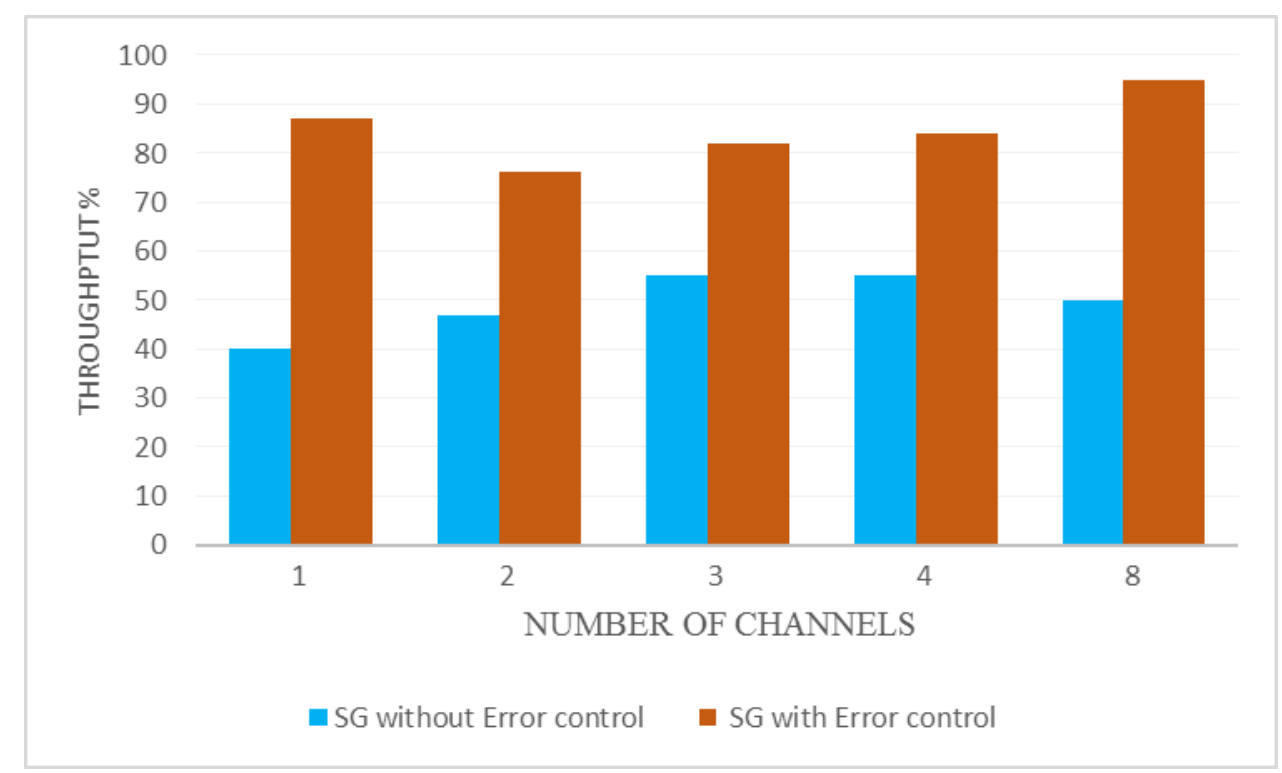


Journal of Electrical Engineering and Automation (EEA) (2019)

Vol.01/ No. 01

Pages: $1-11$

https://www.irojournals.com/iroeea

DOI: https://doi.org/10.36548/jeea.2019.1.001

Fig. 5 Throughput

The fig .5 shows the throughput achieved for different number of channels, for the channels with and without the error control mechanism, the results obtained shows that the throughput achieved through the error control method is very much enhanced compared to the channel without error control mechanism.

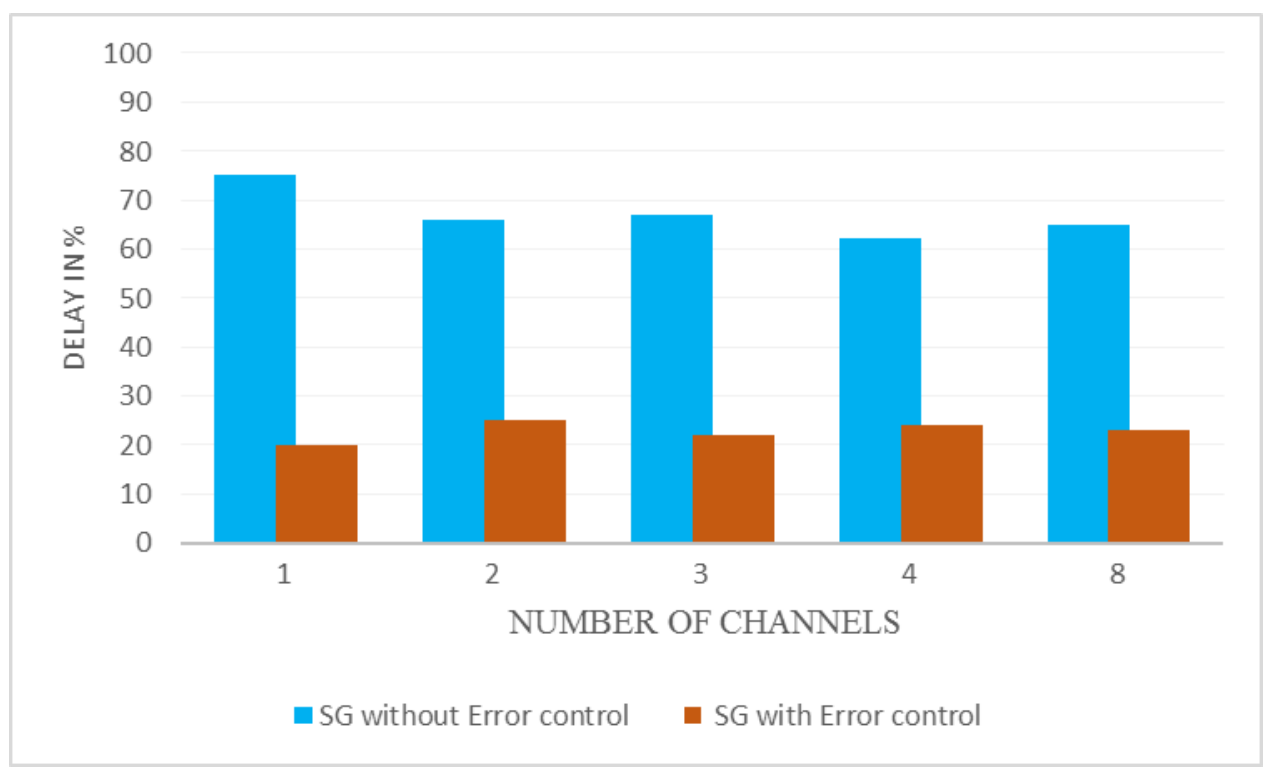

Fig .6 percentage of Delay Incurred

The fig 6 gives the delay incurred in the channel due to the transmission though the error control mechanism consumes a certain level of time for the assigning of the optimal hashes for controlling the error and swapping, this seems to very much less compared to the delay incurred in the channels without the error control mechanism due to the loss in the path and the noise occurrence.

\section{CONCLUSION}

The paper focusing on the security issues arising in the smart grid, for the communication of the information gathered based on the monitoring employing a smart monitoring, handles the issue by employing an error control algorithm that is adaptive to the continuous alterations in the behavior of the channels. The algorithm based on the 
Journal of Electrical Engineering and Automation (EEA) (2019)

Vol.01/ No. 01

Pages: $1-11$

https://www.irojournals.com/iroeea

DOI: https://doi.org/10.36548/jeea.2019.1.001

hash Reed -Solomon is utilized to control the error in the smart grid system and make it proficient. The further results obtained based on the bits error rate, delay and throughput utilizing various modulation techniques evinces the error control adopted by the proposed method improving the efficiency of the smart grid.

\section{References}

[1] Bu, Shengrong, F. Richard Yu, Yegui Cai, and Xiaoping P. Liu. "When the smart grid meets energyefficient communications: Green wireless cellular networks powered by the smart grid." IEEE Transactions on Wireless Communications 11, no. 8 (2012): 3014-3024.

[2] Erol-Kantarci, Melike, and Hussein T. Mouftah. "Energy-efficient information and communication infrastructures in the smart grid: A survey on interactions and open issues." IEEE Communications Surveys \& Tutorials 17, no. 1 (2014): 179-197.

[3] Farhangi, Hassan. "The path of the smart grid." IEEE power and energy magazine 8, no. 1 (2009): 18-28.

[4] Fang, Xi, Satyajayant Misra, Guoliang Xue, and Dejun Yang. "Smart grid-The new and improved power grid: A survey." IEEE communications surveys \& tutorials 14, no. 4 (2011): 944-980.

[5] Amin, S. Massoud, and Bruce F. Wollenberg. "Toward a smart grid: power delivery for the 21st century." IEEE power and energy magazine 3, no. 5 (2005): 34-41.

[6] Ipakchi, Ali, and Farrokh Albuyeh. "Grid of the future." IEEE power and energy magazine 7, no. 2 (2009): $52-62$.

[7] Gungor, Vehbi C., Dilan Sahin, Taskin Kocak, Salih Ergut, Concettina Buccella, Carlo Cecati, and Gerhard P. Hancke. "Smart grid technologies: Communication technologies and standards." IEEE transactions on Industrial informatics 7, no. 4 (2011): 529-539.

[8] Tuballa, Maria Lorena, and Michael Lochinvar Abundo. "A review of the development of Smart Grid technologies." Renewable and Sustainable Energy Reviews 59 (2016): 710-725.

[9] Kabalci, Yasin. "A survey on smart metering and smart grid communication." Renewable and Sustainable Energy Reviews 57 (2016): 302-318.

[10] Kim, Yocheol, Jung Nam Bae, and Jin Young Kim. "Performance of power line communication systems with noise reduction scheme for smart grid applications." IEEE Transactions on Consumer Electronics 57, no. 1 (2011): 46-52.

[11]Fadel, Etimad, Vehbi C. Gungor, Laila Nassef, Nadine Akkari, MG Abbas Malik, Suleiman Almasri, and Ian F. Akyildiz. "A survey on wireless sensor networks for smart grid." Computer Communications 71 (2015): 22-33. 
Journal of Electrical Engineering and Automation (EEA) (2019)

Vol.01/ No. 01

Pages: $1-11$

https://www.irojournals.com/iroeea

DOI: https://doi.org/10.36548/jeea.2019.1.001

[12] Kim, Yo Cheol, Jung Nam Bae, and Jin Young Kim. "Novel noise reduction scheme for power line communication systems with smart grid applications." In 2011 IEEE International Conference on Consumer Electronics (ICCE), pp. 791-792. IEEE, 2011.

[13] Narendran, B., James Sienicki, Shalini Yajnik, and Prathima Agrawal. "Evaluation of an adaptive power and error control algorithm for wireless systems." In Proceedings of ICC'97-International Conference on Communications, vol. 1, pp. 349-355. IEEE, 1997. 